\title{
Discriminatory Proofreading Regimes in Nonequilibrium Systems
}

\author{
Arvind Murugan, ${ }^{1,2,}$ David A Huse, ${ }^{3}$ and Stanislas Leibler ${ }^{1,4}$ \\ ${ }^{1}$ Simons Center for Systems Biology, School of Natural Sciences, \\ Institute for Advanced Study, Princeton, New Jersey 08540, USA \\ ${ }^{2}$ SEAS, Harvard University, Cambridge, Massachusetts 02138, USA \\ ${ }^{3}$ Physics Department, Princeton University, Princeton, New Jersey 08544, USA \\ ${ }^{4}$ Laboratory of Living Matter, The Rockefeller University, \\ 1230 York Avenue, New York, New York 10065, USA
}

(Received 1 November 2013; revised manuscript received 2 February 2014; published 25 April 2014)

We use ideas from kinetic proofreading, an error-correcting mechanism in biology, to identify new kinetic regimes in nonequilibrium systems. These regimes are defined by the sensitivity of the occupancy of a state of the system to a change in its energy. In biological contexts, higher sensitivity corresponds to stronger discrimination between molecular substrates with different energetics competing in the same reaction. We study this discriminatory ability in systems with discrete states that are connected by a general network of transitions. We find multiple regimes of different discriminatory ability when the energy of a given state of the network is varied. Interestingly, the occupancy of the state can even increase with its energy, corresponding to an "antiproofreading" regime. The number and properties of such discriminatory regimes are limited by the topology of the network. Finally, we find that discriminatory regimes can be changed without modifying any "hard-wired" structural aspects of the system but rather by simply changing external chemical potentials.

DOI: 10.1103/PhysRevX.4.021016

\section{INTRODUCTION}

Nonequilibrium systems often show varied behavior that depends on many parameters and details of the system. While it may be easy to investigate any particular point in the parameter space of a given system, it is harder to identify qualitative regimes relevant across systems. For example, the occupancy of states at thermal equilibrium is given simply by the Boltzman distribution $\psi_{\mathrm{eq}}(E) \sim e^{-E / k_{B} T}$, which depends only on the energy $E$ of a state and the temperature $T$. When driven out of equilibrium, the occupancy of states could, in principle, depend on all of the details of the space of the states of the system and the network of paths connecting them. There has been much effort invested [1-4] in understanding nonequilibrium steady states (their occupancy of states, fluxes, and entropy production) in order to model the many systems in physics [5-10] and biology [11-16] that operate out of equilibrium.

In this paper, we identify steady-state kinetic regimes that can be exhibited by general systems that are driven out of equilibrium. These regimes are characterized by the

\footnotetext{
* Corresponding author. amurugan@seas.harvard.edu
}

Published by the American Physical Society under the terms of the Creative Commons Attribution 3.0 License. Further distribution of this work must maintain attribution to the author(s) and the published article's title, journal citation, and DOI.
Subject Areas: Biological Physics, Materials Science, Statistical Physics

change in the occupancy of a state in response to a change in its energy, with all driving forces held fixed.

We draw on intuition from generalized versions of a biological error-correcting mechanism called kinetic proofreading. In biochemical contexts, two molecular substrates, one of them undesirable, might compete to participate in the same enzymatic reaction with different binding energies. Hopfield [17] and Ninio [18] proposed a nonequilibrium "kinetic proofreading" mechanism that enhances the effect of the binding energy difference on reaction rates. As a result, reactions with the weakly binding substrate are suppressed to a much larger extent than one would expect at equilibrium. Kinetic proofreading has been evoked to explain very low error rates in a host of biochemical reactions despite the similarity of competing substrates: in DNA replication, despite the similarity of the four nucleic acids; in protein synthesis despite the similarity of t-RNA molecules [17-20]; in an immune response that discriminates between native and foreign proteins [21]; and in many other biological phenomena [13,15,22,23]. In all of these cases, proofreading enhances discrimination between competing substrates by making the occupancy of a biochemical state more sensitive to changes in its energy.

The central proposition of our paper is that nonequilibrium systems such as proofreading mechanisms can be designed to have coexisting regimes of varying discrimination. In the context of proofreading, some undesirable substrates can be highly discriminated against while other 
substrates of even weaker binding energy can actually be promoted. The number and properties of such coexisting discriminatory regimes are constrained by the topology of the network of paths connecting the system's states. Within these constraints, we can design variable discrimination through an appropriate choice of kinetic parameters that might be "hard wired," for example, in the structural and dynamical properties of molecules.

On the other hand, nonequilibrium steady states are always powered by external sources of free energy, such as Adenosine Tri-Phosphate (ATP) hydrolysis in the case of proofreading. We find that merely changing the chemical potential of these external sources can change the discrimination characteristics. The ability to tune such characteristics on the fly, without having to change any hard-wired properties, raises interesting ways in which natural and synthetic systems can respond to the environment.

\section{ONE-LOOP NETWORK}

We can illustrate many of our results using the simple three-state system shown in Fig. 1(a), with stochastic transitions governed by kinetic rates $k_{i j}$. We then generalize these results to nonequilibrium networks with many more states and pathways (Fig. 2). The model shown in Fig. 1(a) was introduced by Hopfield [17] and Ninio [18] as a nonequilibrium solution to the following discrimination problem in biochemistry: Enzyme $E$ reacts with a substrate $R$, forms complexes $E R, E R^{*}$, and thus processes $R$ into a biologically active product ( $R$ product). However, a structurally similar competing substrate $W$ might also be able to undergo the same set of reactions with $E$, with less favorable energetics, releasing an undesirable product $W$ product.

The steady-state occupancies $\psi_{E R}, \psi_{E R *}, \psi_{E}$ are given by solving the master equation,

$$
\partial_{t} \psi_{i}=\sum_{j} w_{j i}^{R} \psi_{j}-w_{i j}^{R} \psi_{i}=0,
$$

where $w_{i j}^{R}$ are the kinetic constants $k_{i j}$ shown on the network on the left in Fig. 1(a). Occupancies $\psi_{E W}$, $\psi_{E W *}, \psi_{E}$ of the $W$ network are given by solving the same equation with modified kinetic constants $w_{i j}^{W}$ shown on the right in Fig. 1(a). The kinetics of $W$ and $R$ differ because the binding energy of $E W$ is lower than $E R$ by $\Delta$; hence, the off rates $w_{21}^{W}, w_{31}^{W}$ are higher than the corresponding rates for $R$ by a factor of $e^{\Delta}$. We do not consider the case of discrimination due to variable activation barriers studied in Refs. [24,25].

The error rate is the ratio of $W$ product to $R$ product formation, given (in the limit of slow formation rate $f$ from the complexes $E W, E R$ ) by [17]

$$
\eta(\Delta)=\frac{\psi_{E W}}{\psi_{E}}(\Delta) \frac{\psi_{E}}{\psi_{E R}} .
$$

If the system is at equilibrium and not driven, the rates $w_{i j}^{R}$, $w_{i j}^{W}$ will both satisfy detailed balance: we can write $w_{i j}^{S} / w_{j i}^{S}=e^{-\left(V_{j}^{S}-V_{i}^{S}\right)}$, where $S=R$ or $W$ and $V_{i}^{S}$ is the energy of state $i$. (We have set $\beta=1 / k_{B} T=1$.) It is easy to see that $\eta(\Delta)=e^{-\Delta}$, the Boltzmann distribution.

Physically, the error rate $\eta(\Delta)$ is a measure of how much the occupancy $\psi_{E R}$ of state $E R$ changes when the energy of $E R$ is raised by $\Delta$, with external driving forces $\mu_{i j}$ held fixed. A larger change in occupancy implies higher discriminatory power between substrates in the biochemical reaction. Hence, we find it useful to define a local measure of this discriminatory ability, the discriminatory index:

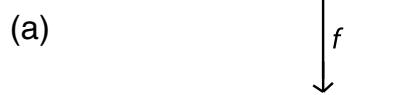

$R$ Product $+E$

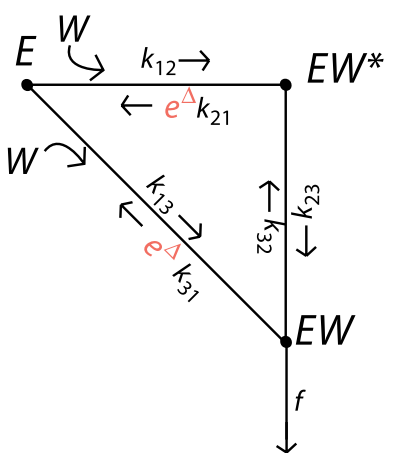

$W$ Product $+E$

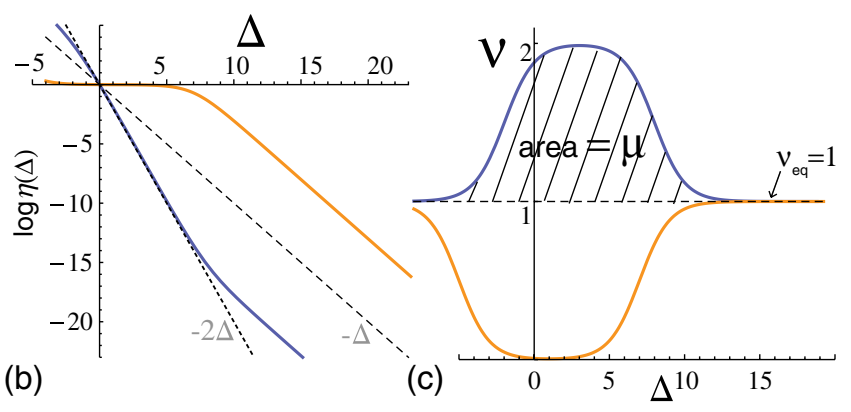

FIG. 1. (a) The kinetic proofreading scheme of Hopfield [17] and Ninio [18] shows variable discrimination between two substrates $W$ and $R$ that compete to participate in the same reactions with an enzyme $E$ but with binding energies that differ by $\Delta$. (b) The ratio $\eta(\Delta)$ of the $W$ product to the $R$ product, with kinetic parameters in the limit proposed by Refs. [17,18], is shown in blue. While a substrate $W$ is suppressed to the extent of $e^{-2 \Delta}$ for $\Delta<8 k_{B} T$, proofreading is less effective for larger $\Delta$. The orange curve in (b) corresponds an alternative limit of kinetics, distinct from that in Refs. [17,18]. Instead of correcting errors, the nonequilibrium network promotes the formation of the less stable $W$ product for $\Delta<6 k_{B} T$, with $\eta(\Delta) \sim 1$. (c) The discriminatory index $\nu$ [i.e., slope of $\log (\eta)]$ shows distinctive discriminatory regimes for both limits of kinetics. The area between the graph of $\nu$ and the equilibrium value $\nu_{\text {eq }}=1$ is equal to the chemical potential $\mu$ used to drive the network. (The area of the orange curve is interpreted as a negative number.) 


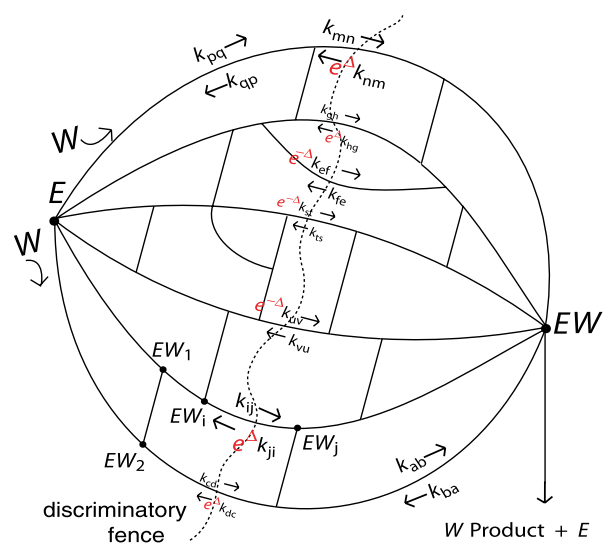

FIG. 2. A general proofreading network with multiple paths from $E+W$ to $E W$. We have shown only the kinetics for substrate $W$. The kinetics $k_{i j}$ for $R$ are identical to those for $W$. except along a set of $c=7$ reactions that cross a "discriminatory fence" (dotted line) and account for the binding energy difference $\Delta$ between $E W$ and $E R$. Reactions on either side of the fence do not discriminate between $W$ and $R$. When driven out of equilibrium, the occupancy of $E W$ can be made as low as $\eta(\Delta) \sim e^{-7 \Delta}$ or as high as $e^{5 \Delta}$ (relative to $E R$ ).

$$
\nu(\Delta) \equiv-\frac{\partial \log \eta(\Delta)}{\partial \Delta}
$$

At equilibrium, $\nu(\Delta)=1$, since $\eta(\Delta)=e^{-\Delta}$.

Hopfield and Ninio proposed that discrimination between $R$ and $W$ can be much higher if the system is driven out of equilibrium by coupling some of the reactions to ATP hydrolysis. The rates $w_{i j}^{S}$, which take ATP coupling into account, will no longer obey detailed balance but can be written as

$$
\frac{w_{i j}^{S}}{w_{j i}^{S}}=e^{-\left(V_{j}^{S}-V_{i}^{S}\right)+\mu_{i j}}
$$

Here, $S=R$ or $W . \mu_{i j}$ is the external driving potential on the $i \leftrightarrow j$ reaction due to ATP coupling and is conservatively assumed to not distinguish between $W$ and $R$. Hopfield and Ninio identified a particular limit of kinetics $w_{i j}^{R}, w_{i j}^{W}$ in which such driving would result in an error rate $\eta \sim e^{-2 \Delta}$ that is typically significantly lower than the equilibrium error rate $e^{-\Delta}$. This mechanism has been invoked to explain low error rates in biochemical processes such as protein synthesis [26]. The general expression for $\eta(\Delta)$ for the Hopfield-Ninio scheme in Fig. 1 is

$$
\eta=\frac{g x\left[1+\left(1+\theta_{1}\right) \theta_{2} x\right]}{1+\theta_{2}\left[1+\theta_{1} \gamma^{-1}(1+\rho)\right] x+\theta_{2}^{2} \theta_{1} \gamma^{-1} \rho x^{2}}
$$

using the definitions $x \equiv e^{-\Delta}$ and

$$
\begin{array}{rlrl}
\theta_{1} & =\frac{k_{12}}{k_{13}}, & \theta_{2}=\frac{k_{23}}{k_{21}}, & \theta_{3}=\frac{k_{31}}{k_{32}}, \\
\rho & =\frac{f}{k_{32}}, & \gamma=\theta_{1} \theta_{2} \theta_{3},
\end{array}
$$

where $g$ is a constant independent of $\Delta$ such that $\eta(0)=1$. Note that $\log \gamma=\mu$ is the chemical potential of the external out-of-equilibrium reaction that powers the proofreading.

Hopfield and Ninio argued that $\eta \sim e^{-2 \Delta}$ in the kinetic limit:

$$
\begin{aligned}
\left(1+\theta_{1}\right) \theta_{2} x & \gg 1, \\
\theta_{2}\left(1+\theta_{1} \gamma^{-1}(1+\rho)\right) x & \ll 1, \\
\theta_{2}^{2} \theta_{1} \gamma^{-1} \rho x^{2} & \ll 1 .
\end{aligned}
$$

We plotted the blue curves in Figs. 1(b) and 1(c) using the following kinetic values that satisfy the above limits:

$$
\gamma=e^{14}, \quad \theta_{1}=e^{10}, \quad \theta_{2}=e^{-2}, \quad \theta_{3}=e^{6}, \quad \rho=e^{-20} .
$$

Discriminatory regimes. - The central observation of our paper is that $\eta$ and $\nu$ are not constant but rather functions of $\Delta$. In particular, the limits in Eq. (6) depend on $x=e^{-\Delta}$ and can be satisfied only for a range of $\Delta$.

The full plot of $\log \eta(\Delta)$ (blue curve in Fig. 1) shows that the classical result of $\eta \sim e^{-2 \Delta}$ holds only for $\Delta<8 k_{B} T$ (in this example), after which $\log \eta(\Delta)$ quickly transitions to a different regime with slope -1 (the equilibrium value). Proofreading is less effective for substrates with $\Delta$ in this regime.

We say that the network exhibits two coexisting "discriminatory regimes," i.e., two distinct regions of $\Delta$ characterized by $\nu \approx 2$ and $\nu \approx 1$, respectively.

Malleability of regimes. - If we choose the alternative set of kinetic constants $k_{i j}$,

$$
\begin{aligned}
& \gamma=e^{-12}, \quad \theta_{1}=e^{10}, \quad \theta_{2}=e^{-15}, \\
& \theta_{3}=e^{-7}, \quad \rho=e^{-20} \text {, }
\end{aligned}
$$

which do not obey Eq. (6) for any $\Delta$, we find a qualitatively different set of regimes with $\nu \approx 0$ and $\nu \approx 1$, shown in Figs. 1(b) and 1(c) (orange curves).

In this sense, discriminatory regimes are malleable and can be changed through an appropriate choice of kinetic constants. (See the Supplemental Material [36] for a 2-loop network example.)

Antiproofreading.-The kinetic limit corresponding to $\nu<1$ [Fig. 1(c), orange curve] uses nonequilibrium effects to actually lower the discrimination between $W$ and $R$; the weakly binding substrate $W$ with $\Delta<6 k_{B} T$ forms as much product as strongly binding $R$ since $\nu \approx 0$ and $\eta(\Delta) \sim 1$. 
This is an example of an "antiproofreading" regime, in which higher energy states can have higher occupancy.

Size of regimes and chemical potentials.-The size of the discriminatory regimes shown in Fig. 1(c) can be quantified by the area between the graph of $\nu(\Delta)$ and the equilibrium value $\nu_{\mathrm{eq}}=1$. This area is, in fact, equal to the net chemical potential around the loop $\mu=\mu_{12}+\mu_{23}+\mu_{31}$ that is used to drive the system. Thus, higher chemical potentials are needed to enhance discrimination across a wider range of energies.

External shaping of regimes.-The relationship between chemical potential and the shape of $\eta(\Delta)$ and $\nu(\Delta)$ implies that we can modify discriminatory regimes without having to change any kinetic constants hard wired in the structure of the enzyme. For example, we can switch from the kinetics corresponding to the blue curve in Fig. 1(c) to kinetics corresponding to the orange curve by flipping the sign of chemical potentials $\mu_{i j}$-i.e., by driving the system in the reverse direction around the reaction loop.

\section{GENERAL NETWORKS}

We now show how the kinetics of a general driven network can give rise to the variable discriminatory regimes discussed above. We find that the topology of the network limits the number, size, and index $\nu$ of discriminatory regimes that can be achieved.

A general driven network with multiple interconnected pathways is shown in Fig. 2. We have only shown the kinetics for substrate $W$, whose binding energy to $E$ is lower than that for $R$ by $\Delta$. This binding energy difference can be reflected in the kinetics in many different ways. The only requirement is that the equilibrium constant computed along any path from start $(E+S)$ to finish $(E S)$ for $S=R$ and $S=W$ must differ by a factor of $e^{\Delta}$. For now, we assume that the distinction in kinetics is localized in one reaction along each pathway, as shown in Fig. 2. We connect such reactions by an imaginary line that we call the "discriminatory fence" (dotted line in Fig. 2), which divides the network in two. Reactions on either side of the fence do not discriminate between the two substrates; the binding energy difference $\Delta$ is entirely accounted for by reactions that cross the discriminatory fence. (The fence is a surface for nonplanar networks. See Supplemental Material [36] for a discussion.)

Discrimination in several biological proofreading mechanisms does appear to be localized in select reactions [26-28]. We relax this simplifying assumption later and find that a delocalized fence lowers the discriminatory ability of the network.

\section{A. Limit of highest discrimination}

To gain intuition about general networks, we begin with the kinetic limit that leads to the highest possible discriminatory index $\nu>0$ (strongest proofreading) in any network. This kinetic limit generalizes several particular models of biological error correction [15,27-30] and is defined by three properties.

(i) The kinetics pick out a dominant path, shown in green in Fig. 3(a), from state $E+S$ to the final state $E S$; the system can reach $E S$ only by traversing this path.

(ii) The dominant path must parallel the discriminatory fence on the products (i.e., $E S$ ) side of the network before reaching $E S$. As a result, as the system travels down the dominant path towards $E S$, it is constantly exposed to

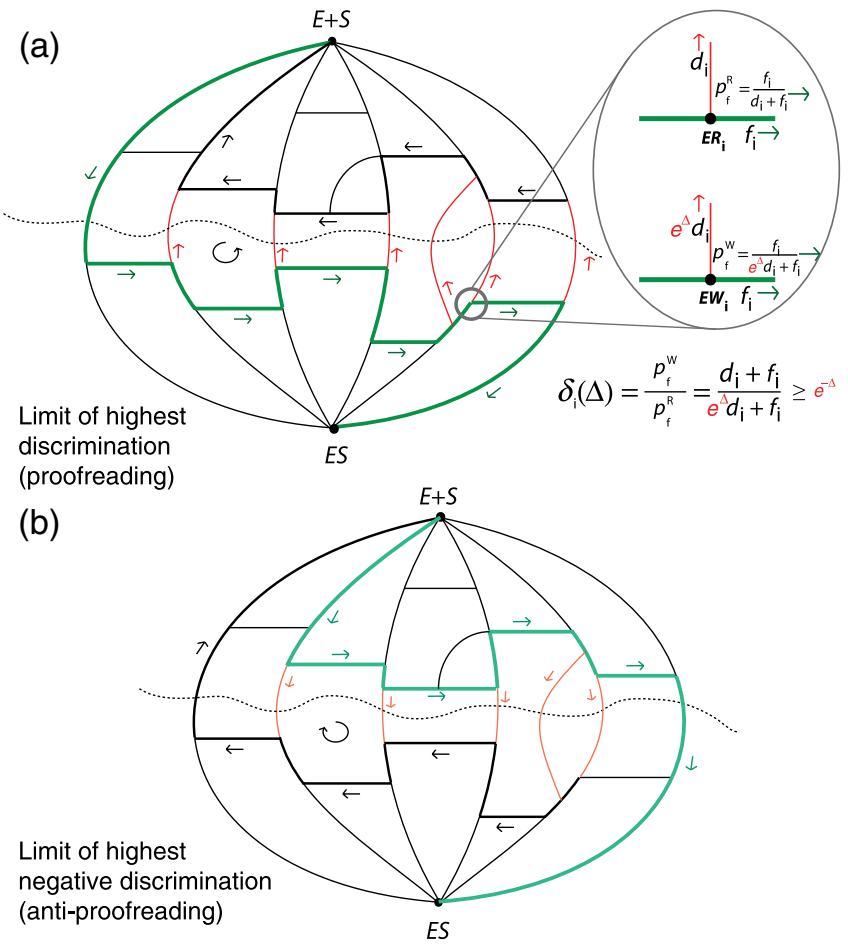

FIG. 3. General limit of kinetics for highest (a) discrimination $\nu>0$ and (b) negative discrimination $\nu<0$ (antiproofreading). In both cases, the system can reach $E S$ only by traveling down a dominant path (green) that parallels the discriminatory fence (dotted line). In this way, the system is exposed to multiple discard pathways (shown in red) that cross the discriminatory fence (dotted line) and abort the reaction by taking the system back to the initial state $E+S$ through the bold black path (here, $S=R$ or $W$ ). (a) As shown in the inset, the probability $p_{f}^{S}$ of not taking a red discard path across the fence is lower for $S=W$ than for $S=R$ by a factor of $\delta_{i}(\Delta)$. With $c$ such discard paths along the dominant path, $W$ is exponentially less likely to reach $E S$ than $R$, resulting in $\eta(\Delta) \sim e^{-c \Delta}$. In (b), the dominant path parallels the fence on the reactants $E+S$ side of the network. The system can now take the (red) discard paths from the reactants side to the product side. Unlike in (a), we allow for the system to possibly bounce back to the dominant path after a discard. As shown in the Supplemental Material [36], $W$ is more likely to bounce back and proceed farther along the dominant (green) path by a factor $\delta_{i}(\Delta)>1$. Hence, the weakly binding $S=W$ substrate is exponentially more likely to reach $E S$. 
pathways (shown in red) that cross the fence back to the reactants $(E+S)$ side.

(iii) The kinetics need to ensure that, if the system crosses the fence to the reactants side along a (red) discard pathway, the system moves back towards $E+S$ along a path like that shown in bold. Hence, we refer to the (red) pathways as discard pathways since they stop progress along the dominant path.

Hence, a typical trajectory in Fig. 3(a) involves the system attempting to traverse the green path to the final state $E S$ but being frequently discarded through the red fence-crossing reactions. The kinetics described thus far apply to both $S=R$ and $S=W$ substrates. The only distinction is that $W$ is more likely to take each discard pathway across the discriminatory fence. To see this, note that the probability of being discarded is determined by the kinetic constants $d_{i}$ for the discard pathway and $f_{i}$ for the forward direction on the dominant pathway [see inset of Fig. 3(a)]. Since $d_{i}$ is higher for $W$ than for $R$ by a factor $e^{\Delta}$, $W$ is less likely to proceed forward along the dominant path by a factor $\delta_{i}$, as shown in Fig. 3(a).

In the limit of high discards $d_{i} \gg f_{i}$, the ratio of the forward probabilities $\delta_{i}$ is $e^{-\Delta}$. If there are $c$ such discard paths branching off the dominant path, the net probability of reaching $E S$ is lower for $W$ than for $R$ by a factor of $\eta(\Delta) \sim e^{-c \Delta}$, giving the highest possible discriminatory index $\nu=c$.

We note that much time is wasted in this limit since both $R$ and $W$ are frequently discarded along the dominant path and reaction completion is an exponentially unlikely event. Energy is also wasted since the system preferentially executes counterclockwise trajectories in the network shown in Fig. 3; such preferential cycling breaks detailed balance and consumes free energy from an external source (such as ATP hydrolysis) during each cycle. An alternative kinetic regime that trades a small increase in error for a large saving in time and energy was found in Ref. [31].

Antiproofreading.-If the dominant path parallels the discriminatory fence on the reactants $(E+S)$ side instead [Fig. 3(b), we find a counterintuitive antiproofreading regime with $\nu<0$ and $\eta \sim e^{+(c-2) \Delta}$. The discard pathways now cross the fence from the reactants to the product side. Unlike in the proofreading case, we assume that there is a high chance of reversing the discard reaction immediately. As a result, the system bounces back and forth repeatedly along a discard path before either proceeding forward along the dominant path or going back to the initial state along the bold black path in Fig. 3(b). As shown in the Supplemental Material [36], substrate $W$ is more likely to bounce back across the fence and resume progress along the dominant path by a factor of $\delta_{i}(\Delta)>1$. Hence, the weakly binding substrate $W$ reaches the final state $E W$ more often than $R$ reaches $E R$. In other words, the occupancy of the state $E S$ increases as its energy is raised.

\section{B. Sculpting multiple discriminatory regimes}

The kinetic pathways for highest positive and negative discrimination suggest how variable discriminatory regimes arise for a general network. As shown in the inset of Fig. 3 for the case of positive discrimination, and in the Supplemental Material for antiproofreading, each $\delta_{i}(\Delta)=$ $\left(d_{i}+f_{i}\right) /\left(d_{i} e^{ \pm \Delta}+f_{i}\right)$ provides varying discrimination that depends on $\Delta$ and the discard $d_{i}$ and forward $f_{i}$ kinetics at that point. In the limits described in Fig. 3, the occupancy ratio $\eta(\Delta)$ is (approximately) a product of $\delta_{i}$ along the dominant path:

$$
\eta(\Delta) \sim \delta_{1}(\Delta) \delta_{2}(\Delta) \ldots \delta_{c}(\Delta)
$$

As a result, we can design a large network to show multiple discriminatory regimes like that shown in Fig. 4(a) through a choice of dominant path and a choice of $\delta_{i} \mathrm{~s}$ along the path. In Fig. 4(b), the dominant path switches midway from paralleling the discriminatory fence on the reactants side [as in Fig. 3(b)] to the products side [as in Fig. 3(a)], allowing a more general product of $\delta_{i}$.

More generally, $\eta$ is a ratio of polynomials $p\left(e^{-\Delta}\right) /$ $q\left(e^{-\Delta}\right)$ in $e^{-\Delta}$,
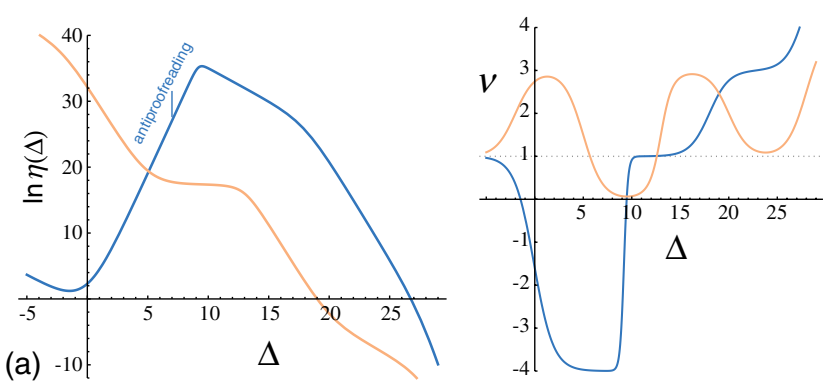

(b)

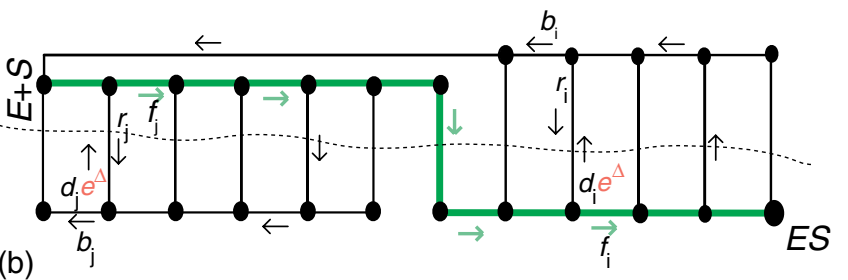

FIG. 4. (a) We can sculpt $\eta(\Delta), \nu(\Delta)$ shown, with multiple discriminatory regimes, by combining the two limits shown in Fig. 3. We use a ladderlike network shown in (b) and a dominant path that switches midway from paralleling the fence on the reactants side [as in Fig. 3(b)] to the products side [as in Fig. 3(a)]. We position the regimes as shown (blue and orange plots) through two different choices of kinetic constants $d_{i}, f_{i}, r_{i}, b_{i}$. These constants determine $\delta_{i}(\Delta)$ [see Fig. 3 and Eq. (9)] along the dominant path. [See the Supplemental Material [36] for numerical values of $d_{i}, f_{i}$ and more on $\delta_{i}(\Delta)$. We added a constant to the orange curve in (a) to fit both curves in the same plot.] 


$$
\eta(x)=x \frac{n_{0}+n_{1} x+\cdots+n_{a} x^{a}}{d_{0}+d_{1} x+\cdots+d_{b} x^{b}} \frac{d_{0}+d_{1}+\cdots+d_{b}}{n_{0}+n_{1}+\cdots+n_{a}},
$$

where $x \equiv e^{-\Delta}$. A discriminatory regime with index $\nu=$ $i-j$ corresponds to a finite range of $\Delta$ over which $\eta(\Delta)$ can be approximated by $a_{i} e^{-i \Delta} / b_{j} e^{-j \Delta}$, where $a_{i} e^{-i \Delta}$ and $b_{j} e^{-j \Delta}$ are particular monomials in $p$ and $q$, respectively. (See the Supplemental Material for a rigorous derivation of these results using Schnakenberg's network theory [5].)

\section{Network topology constrains regimes}

With $\eta\left(e^{-\Delta}\right)=p\left(e^{-\Delta}\right) / q\left(e^{-\Delta}\right)$, we find that the degrees of the polynomials $p, q$ are bound by $c$, the number of intersections between the discriminatory fence and the network. As a result, topological properties of the proofreading reaction network constrain the shape of $\eta(\Delta)$ and index $\nu(\Delta)$.

(i) The largest positive and negative values of $\nu$ are limited by $c:-(c-2)<\nu<c$.

(ii) The number of distinct discriminatory regimesdetermined by the number of monomials in $p, q-$ is also bounded by $c$.

(iii) The extremal values attained by $\nu$ over all $\Delta$ are related by $\nu_{\max }-\nu_{\min } \leq c$.

(iv) The intersection number $c$ cannot exceed the number of linearly independent pathways. Equivalently, in terms of the number of linearly independent loops $n$ in the network, $c \leq n+1$.

(v) This sets an absolute limit on the discrimination between two substrates whose binding energies differ by $\Delta$ by a proofreading network with $n$ loops:

$$
e^{(n-1) \Delta} \geq \eta(\Delta) \geq e^{-(n+1) \Delta} .
$$

If the discriminatory fence intersects only $c<n+1$ reactions, then $e^{(c-2) \Delta} \geq \psi(\Delta) \geq e^{-c \Delta}$. For the network shown in Fig. 2, $c=7$ while $n=18$.

We find that the minimal chemical potentials required for a discriminatory index $\nu(\Delta)$ are given by the areas of the $n$ discriminatory regimes in the graph of $\nu-1$. When $\nu<1$, the area and chemical potential required are negative, indicating that the corresponding loop must be driven in the opposite direction. We leave a study of the precise mapping between the $n$ linearly independent chemical potentials $\mu_{l}$ in a network with $n$ loops and the $n$ resulting regimes to future work.

\section{Localized discrimination fence and von Neumann's error-correcting scheme}

We have assumed that the binding energy difference $\Delta$ is localized in a set of reactions, one along each pathway from $E+S$ to $E S$, that define the sharp discriminatory fence. Consider the alternatives shown in Fig. 5, where the energy $\Delta$ is [(5b)] spread over multiple reactions along each
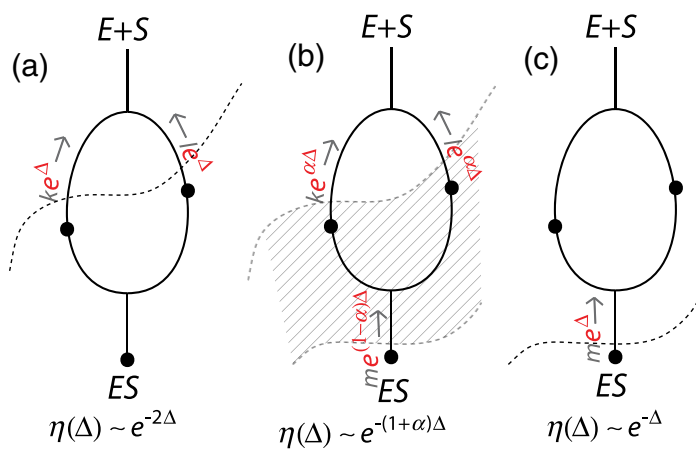

FIG. 5. (a) Discrimination is highest when the energy difference $\Delta$ is localized to a discriminatory fence that cuts through as many reactions as possible. In panels (b) and (c), a fraction $\alpha$ or all of the energy difference $\Delta$ is in reactions common to both pathways $(0 \leq \alpha \leq 1)$. Proofreading acts by multiplying the effect of discrimination in parallel and independent reactions but cannot enhance discriminatory reactions common to both pathways.

pathway or $[5(\mathrm{c})]$ where the energy difference $\Delta$ is localized in a reaction common to both pathways. Proofreading can only combine independent discriminatory power in parallel pathways that form loops. Hence, while the network in Fig. 5(a) achieves discrimination of $e^{-2 \Delta}$, Figs. 5(b) and 5(c) can only achieve lower discrimination of $e^{-(1-\alpha) \Delta} e^{-2 \alpha \Delta}=e^{-(1+\alpha) \Delta}$ and $e^{-\Delta}$, respectively. [In Fig. 5(b), we assume $0 \leq \alpha \leq 1$, so that no individual reaction has discriminatory power greater than $e^{-\Delta}$.]

We can think of proofreading as a biochemical implementation of von Neumann's [32] reliable machine made of redundant unreliable components. von Neumann constructed logical machines that failed only when all of the individual error-prone components failed. Thus, the machine itself has a lower error rate than the individual components, provided the components have independent probabilities of failure. In proofreading, each pathway can complete the entire reaction in isolation with an error rate of $e^{-\Delta}$, but the network as a whole can have a lower error rate $\eta(\Delta) \ll e^{-\Delta}$. In this context, the two pathways of Fig. 5(a) are like von Neumann components with fully independent error rates while the error rates of the pathways in Fig. 5(b) and 5(c) are not independent and cannot be combined as effectively.

\section{DISCUSSION}

By generalizing kinetic proofreading, we have uncovered new kinetic regimes in systems driven out of equilibrium, characterized by how much the occupancy of a state changes due to a change in its energy. The occupancy can fall with energy much faster than at equilibrium (conventional proofreading), but we also find antiproofreading regimes where the occupancy of a state increases with increasing energy. We find bounds on the number, size, and discriminatory ability of regimes in terms of 
topological properties of the network of transitions between states.

We relate discriminatory regimes to kinetic limits involving frequent discards from a dominant path. In earlier work, we pointed out that such kinetics resemble the "dynamic instability" of microtubule growth [31]. Combined with the results in this paper, we can say that discriminatory regimes are related to dynamic instability along a dominant path in a general reaction network. The nature of the regimes is determined by the relative placement of the dominant path and the discriminatory fence (the collection of reactions that account for the change in the energy of a state).

Biologically, having proofreading ability vary with binding energy can be useful when a biochemical reaction is presented with a family of competing substrates. The same proofreading scheme might be able to enhance discrimination between two given substrates while in fact suppressing differences between another pair, depending on their binding energies.

We emphasize that our results apply to networks that represent any driven system, including systems unrelated to biological kinetic proofreading. For example, the states of the network could represent different stages of assembly of microtubules [31] or synthetic self-assembled structures [33-35]. Our results then might point the way to incorporating driving forces into the assembly pathways in synthetic systems, reducing errors in the final structure.

An intriguing feature of the schemes discussed here is the possibility of changing the discriminatory regimes by changing only the chemical potentials $\mu_{i}$, without having to change any hard-wired structural kinetics. Such an ability to change discriminatory properties on the fly could be useful in both natural and synthetic systems; the network can switch between suppressing the reaction with a substrate and promoting it, depending on the environment or other considerations.

\section{ACKNOWLEDGMENTS}

We thank John J. Hopfield and Luca Peliti for stimulating discussions. We are also grateful to Luca Peliti for detailed comments on a draft of this paper.

[1] C. Bustamante, J. Liphardt, and F. Ritort, The Nonequilibrium Thermodynamics of Small Systems, Phys. Today 58, No. 7, 43 (2005).

[2] U. Seifert, Stochastic Thermodynamics: Principles and Perspectives, Eur. Phys. J. B 64, 423 (2008).

[3] D. Q. Jiang, M. Qian, and M. P. Qian, Mathematical Theory of Nonequilibrium Steady States: On the Frontier of Probability and Dynamical Systems, Lecture Notes in Mathematics Vol. 1833 (Springer, New York, 2004).
[4] T. Chou, K. Mallick, and R. K. P. Zia, Nonequilibrium Statistical Mechanics: From a Paradigmatic Model to Biological Transport, Rep. Prog. Phys. 74, 116601 (2011).

[5] J. Schnakenberg, Network Theory of Microscopic and Macroscopic Behavior of Master Equation Systems, Rev. Mod. Phys. 48, 571 (1976).

[6] R. K. P. Zia and B. Schmittmann, A Possible Classification of Nonequilibrium Steady States, J. Phys. A 39, L407 (2006).

[7] R. K. P. Zia and B. Schmittmann, Probability Currents as Principal Characteristics in the Statistical Mechanics of Nonequilibrium Steady States, J. Stat. Mech. (2007) P07012.

[8] B. Derrida, Nonequilibrium Steady States: Fluctuations and Large Deviations of the Density and of the Current, J. Stat. Mech. (2007) P07023.

[9] T. Schmiedl and U. Seifert, Stochastic Thermodynamics of Chemical Reaction Networks, J. Chem. Phys. 126, 044101 (2007).

[10] S. Vaikuntanathan, T. R. Gingrich, and P. L. Geissler, Dynamic Phase Transitions in Simple Driven Kinetic Networks, arXiv:1307.0801.

[11] T. L. Hill, Free-Energy Transduction and Biochemical Cycle Kinetics (Dover, Mineola, NY, 1989).

[12] H. Qian, Cycle Kinetics, Steady-State Thermodynamics, and Motors: A Paradigm for Living Matter Physics, J. Phys. Condens. Matter 17, S3783 (2005).

[13] H. Qian, Open-System Nonequilibrium Steady State: Statistical Thermodynamics, Fluctuations, and Chemical Oscillations, J. Phys. Chem. B 110, 15063 (2006).

[14] U. Seifert, Stochastic Thermodynamics of Single Enzymes and Molecular Motors, Eur. Phys. J. E 34, 1 (2011).

[15] Y. Tu, The Nonequilibrium Mechanism for Ultrasensitivity in a Biological Switch: Sensing by Maxwell's Demons, Proc. Natl. Acad. Sci. U.S.A. 105, 11737 (2008).

[16] G. Lan, P. Sartori, S. Neumann, V. Sourjik, and Y. Tu, The Energy-Speed-Accuracy Trade-Off in Sensory Adaptation, Nat. Phys. 8, 422 (2012).

[17] J. J. Hopfield, Kinetic Proofreading: A New Mechanism for Reducing Errors in Biosynthetic Processes Requiring High Specificity, Proc. Natl. Acad. Sci. U.S.A. 71, 4135 (1974).

[18] J. Ninio, Kinetic Amplification of Enzyme Discrimination, Biochimie 57, 587 (1975).

[19] M. Johansson, J. Zhang, and M. Ehrenberg, Genetic Code Translation Displays a Linear Trade-Off between Efficiency and Accuracy of tRNA Selection, Proc. Natl. Acad. Sci. U.S.A. 109, 131 (2012).

[20] K. B. Gromadski and M. V. Rodnina, Kinetic Determinants of High-Fidelity tRNA Discrimination on the Ribosome, Mol. Cell 13, 191 (2004).

[21] T. W. McKeithan, Kinetic Proofreading in T-Cell Receptor Signal Transduction, Proc. Natl. Acad. Sci. U.S.A. 92, 5042 (1995).

[22] T. Tlusty, R. Bar-Ziv, and A. Libchaber, High-Fidelity DNA Sensing by Protein Binding Fluctuations, Phys. Rev. Lett. 93, 258103 (2004).

[23] H. Ge, M. Qian, and H. Qian, Stochastic Theory of Nonequilibrium Steady States. Part II: Applications in Chemical Biophysics, Phys. Rep. 510, 87 (2012).

[24] C. H. Bennett, Dissipation-Error Trade-Off in Proofreading, BioSystems 11, 85 (1979). 
[25] P. Sartori and S. Pigolotti, Kinetic versus Energetic Discrimination in Biological Copying, Phys. Rev. Lett. 110, 188101 (2013).

[26] M. Johansson, M. Lovmar, and M. Ehrenberg, Rate and Accuracy of Bacterial Protein Synthesis Revisited, Curr. Opin. Microbiol. 11, 141 (2008).

[27] G. Altan-Bonnet and R. N. Germain, Modeling T-Cell Antigen Discrimination Based on Feedback Control of Digital ERK Responses, PLoS Biol. 3, e356 (2005).

[28] R. Bar-Ziv, T. Tlusty, and A. Libchaber, Protein-DNA Computation by Stochastic Assembly Cascade, Proc. Natl. Acad. Sci. U.S.A. 99, 11589 (2002).

[29] T. W. McKeithan, Kinetic Proofreading in T-Cell Receptor Signal Transduction, Proc. Natl. Acad. Sci. U.S.A. 92, 5042 (1995).

[30] M. A. Savageau, Accuracy of Proofreading with Zero Energy Cost, J. Theor. Biol. 93, 179 (1981).

[31] A. Murugan, D. A. Huse, and S. Leibler, Speed, Dissipation, and Error in Kinetic Proofreading, Proc. Natl. Acad. Sci. U.S.A. 109, 12034 (2012).
[32] J. von Neumann, in Automata Studies, edited by C. Shannon and J. McCarthy (Princeton University Press, Princeton, NJ, 1956).

[33] B. Wei, M. Dai, and P. Yin, Complex Shapes Self-Assembled from Single-Stranded DNA Tiles, Nature (London) 485, 623 (2012).

[34] N. P. King, W. Sheffler, M. R. Sawaya, B. S. Vollmar, J. P. Sumida, I. Andre, T. Gonen, T. O. Yeates, and D. Baker, Computational Design of Self-Assembling Protein Nanomaterials with Atomic Level Accuracy, Science 336, 1171 (2012).

[35] D. Y. Zhang and G. Seelig, Dynamic DNA Nanotechnology Using Strand-Displacement Reactions, Nat. Chem. 3, 103 (2011).

[36] See Supplemental Material at http://link.aps.org/ supplemental/10.1103/PhysRevX.4.021016 for derivations of error rate and the discriminatory index using Schankenberg network theory for arbitrary networks as well as numerical details. 